MS34-P18 Coordination Frameworks of

Tetrakis[meso-(3,5-biscarboxyphenyl)]-Metalloporphyrins

with Polynuclear Metallic Nodes

Israel Goldberg $^{1}$, Bharat Kumar Tripuramallu ${ }^{1}$

1. School of Chemistry, Tel Aviv University

email: goldberg@post.tau.ac.il

Metal organic frameworks deserve increasing attention as an emerging class of porous functional materials in recent years. The most successful design strategy for constructing these crystalline frameworks is to take advantage of the versatile geometry of organic building units (linkers) and metal centers (uni- and multinuclear connecting nodes), which greatly influence the resulting architectures of the formed framework. Tetrapyrrole macrocycles or porphyrin building blocks are quintessential for forming quadrangular open frameworks, owing to their bulkiness and $\mathrm{D}_{4}$ symmetry. Multiple functionality of the organic linkers is important for promoting expansion of the formed framework in three dimensions.

Prior to this study formulation and characterization of octacarboxy porphyrin-based metal organic frameworks have enjoyed a rather slow progress in view of the synthetic difficulty in obtaining sizeable crystalline samples, as polycrystalline or amorphous solids are most frequently the outcome of common preparative procedures. On rare occasions, when small crystallites became available, their structural characterizations by $\mathrm{X}$-ray diffraction technique required access to synchrotron radiation sources due to the weak diffraction power of such crystals and high content of the disordered solvent trapped in the channel-perforated lattice.

This work provides an insight into an improved synthetic methodology of crystalline framework solids with the fast-reacting octa-topic tetrakis(3,5-dicarboxyphenyl) metalloporphyrin linker and a variety of metallic nodes, using $\mathrm{NaOH}$ as a modulator to enhance crystal growth. During the supramolecular reaction the hydroxyl ions supplied by the $\mathrm{NaOH}$ compete with the carboxylate anions for coordinating to the metal (or metal cluster), slowing the reaction rate between the metal ions and the porphyrin, and thus enhancing crystal growth of the solid polymeric product. Here we describe crystalline framework materials of the octa-carboxy porphyrin ligand obtained with a series of metal centers, including lanthanoid and transition metal ions, as $\mathrm{Pr}, \mathrm{Eu}, \mathrm{Gd}, \mathrm{Tb}, \mathrm{Mn}, \mathrm{Fe}, \mathrm{In}$ and $\mathrm{Ga}$, as well as $\mathrm{Na}$ centers. The structural diversity of the framework architectures and of the interaction synthons will be discussed.

B. K. Tripuramallu and I. Goldberg, Cryst. Growth Des. 2016, 16, 1751-1764.

B. K. Tripuramallu, H. M. Titi, S. Roy, R. Verma and I. Goldberg, CrystEngComm 2016, 18, 515-520.

Keywords: metal-organic-frameworks

\section{MS35-P1 Martensitic solid-to-solid transitions in amino acid crystals: what insight can simulations bring to the table? \\ Herma M. Cuppen ${ }^{1}$}

1. Institute for Molecules and Materials, Radboud University

email: h.cuppen@science.ru.nl

Many molecular compounds exhibit polymorphism: the ability to crystallize in different crystal structures. The polymorphic forms determine crystal properties such as the solubility rate, which is key in dose determination in a pharmaceutical context. Understanding of solid-to-solid transitions between different polymorphic forms is the first step in controlling the polymorphic stability of a compound.

Polymorphic transitions are driven by a free energy difference. If the relative stability of different polymorphic forms changes reversibly as a function of temperature, one speaks of enantiotropically related polymorphic forms. In the case of transformations in which the parent and daughter phase have similar structures, there is an ongoing debate whether the transformation mechanism is martensitic or occurs through nucleation and growth. I will present advanced Molecular Dynamics (MD) simulations of two enantiotropically related polymorphic forms of DL-norleucine: $\alpha$ (room temperature) and $\beta$ (low temperature), which are thought to transform through a martensitic mechansism by sliding along two cell axes. I will show that the mechanism of the transition occurs through a cooperative movement of bilayers via an intermediate state $[1,2]$. Although the layers move in a concerted fashion, the results further indicate that local fluctuations in the conformations of the aliphatic chains play a crucial role in keeping the cooperative mechanism sustainable at large length scales (see figure). The results suggest a mechanism where formation of a nucleus of the new phase occurs through cooperative motion, which then grows through propagation in a wave-like manner through the crystal [3]. At the length scale of theinitial size of the cluster, classical nucleation theory and the cooperative mechanism could naturally come together.

References:

[1] J.A. van den Ende and H.M. Cuppen Cryst. Growth Des., 2014, 14, 3343

[2] J. A. van den Ende, M.M.H. Smets, D.T. de Jong, S.J.T. Brugman, B. Ensing, P.T. Tinnemans, H. Meekes, and H. M. Cuppen Faraday Disc., 2015, 179, 421

[3] J. A. van den Ende, B. Ensing, and H. M. Cuppen CrystEngComm, accepted 